\section{PTU-044 HEPATIC/ENDOTHELIAL CELL CO-CULTURE; ESTABLISHING OPTIMAL CONDITIONS FOR LIVER TISSUE ENGINEERING}

doi:10.1136/gutjnl-2012-302514c.44

${ }^{1} \mathrm{M}$ Navarro, ${ }^{1} \mathrm{~L}$ Nelson, ${ }^{2} \mathrm{~K}$ Burgess, ${ }^{3} \mathrm{O}$ Tura, ${ }^{3} \mathrm{~K}$ Samuel, ${ }^{1} \mathrm{~J}$ Plevris. ' Laboratory Department of Hepatology, University of Edinburgh, Edinburgh, UK; ${ }^{2}$ ScotMet: The Scottish Metabolomics Facility, University of Glasgow, Glasgow, UK; ${ }^{3} \mathrm{MRC} / \mathrm{Scottish}$ Centre for Regenerative Medicine, University of Edinburgh, Edinburgh, UK

Introduction Development of 3D hepatic organoids utilising human cell derivatives for in vitro drug testing and bioartificial liver support systems is challenging. Tissue engineering thick, complex structures such as human liver organoids (micro-tissue; $>500 \mathrm{~mm}$ ) will require vascularisation of $3 \mathrm{D}$ cultures-coupled with biomatrix support scaffolds - to both maintain integrity and promote formation of endothelial channels (sinusoid-like structures) to facilitate oxygen and nutrient transfer to hepatocytes in 3D culture. Key challenges involve directing nascent microvessels into an appropriate environment defined prominently by heterotypic cell-cell contacts and meeting high metabolic demands. In this preliminary study, we aimed to optimise heterotypic co-culture of either Human Umbilical Vein Endothelial Cells (HUVECs) or Endothelial Outgrowth $\underline{\text { Cells }}$ (EOCs) with hepatic C3A cells, using appropriate biomatrix scaffolds.

Methods Different ratios of HUVECs: C3A or EOCs:C3A to form co-cultures were studied using contrast/confocal microscopy and flow cytometry following appropriate immunostaining. Flow cytometry was used to study integrin expression (Cd49a, Cd49b, Cd49f, Cd49e), endothelial markers (CD31) and EpCam, as an hepatic marker. Fingerprint unbiased metabolomics analysis was used to assess function of co-cultured cells. Matrigel and MaxGel (Sigma) were tested as candidate bioscaffolds and were compared with standard 2D cultures on plastic and collagen for each cell line as controls.

Results A ratio 3:1 (HUVECs:C3A) was optimal for growth using endothelial culture medium (Lonza EGM-2 medium, UK). Cell phenotype was maintained for 7 days in co-culture with strong integrin (Cd49a, Cd49b, Cd49f, Cd49e), and CD31 expression on HUVECs and EpCam on C3A cells, while a reduction of HUVEC cell number was noted with a parallel increase of C3A cells by day 7-to form more sheet-like structures in co-culture. Metabolomics analysis of culture media showed enhanced urea cycle, lipid synthesis and amino acid utilisation of C3A cells in co-culture. Matrigel promoted formation of microvessel structures, with interconnected channels, in both EOCs and HUVECs; and was superior to MaxGel. MaxGel in 3D sandwich culture promoted differentiated (cuboidal) morphology of C3As, but not EoCs.

Conclusion Optimisation of both cell ratios and cell numbers, as well as selection of appropriate culture media are critical factors in developing a successful hepatic co-culture system with the ability to form sinusoid-like/microvessel structures. This study represents an early step towards understanding the requirements of vascularised liver tissue for future clinical/pharmaceutical applications.

Competing interests None declared.

\section{PTU-045 PROTON NUCLEAR MAGNETIC RESONANCE SPECTROSCOPY OF PLASMA IN PATIENTS WITH CIRRHOSIS CORRELATES WITH ARTERIAL AMIMONIA BUT NOT GRADE OF HEPATIC ENCEPHALOPATHY}

doi:10.1136/gutjnl-2012-302514c.45

II Coltart, ${ }^{1,2} \mathrm{M}$ J W McPhail, ${ }^{*} \mathrm{E} \mathrm{J}$ Want, ${ }^{3} \mathrm{~K}$ Veselkov, ${ }^{3} \mathrm{E}$ Holmes, ${ }^{1} \mathrm{~J}$ Wendon, ${ }^{2} \mathrm{~S}$ Taylor-Robinson, ${ }^{1} \mathrm{~W}$ Bernal, ${ }^{1} \mathrm{D}$ Shawcross. ${ }^{1}$ Institute of Liver Studies, Kings College
Hospital, London, UK; ${ }^{2}$ Liver \& Anti Viral Centre, London, UK; ${ }^{3}$ Biomolecular Medicine, Imperial College London, London, UK

Introduction Diagnosis of overt hepatic encephalopathy (HE) in patients with cirrhosis often relies on subjective clinical examination. Arterial ammonia correlates poorly with HE grades and a blood marker for diagnosis and monitoring is lacking. Metabolic profiling by nuclear magnetic resonance (NMR) spectroscopy of blood correlates with Model for End Stage Liver Disease Score (MELD) ${ }^{1}$ and may offer diagnostic biomarkers in overt HE.

Methods Thirty-seven patients with cirrhosis and differing grades of HE were identified. Arterial blood was drawn for arterial ammonia, blood gas analysis and NMR spectroscopy. HE was diagnosed and graded by West-Haven criteria and Trail Making (A and B) Tests. ${ }^{1} \mathrm{H}$ NMR spectroscopy was performed in a Bruker $600 \mathrm{MHz}$ Avance spectrometer using a Carr-Purcell-Meiboom-Gill sequence prior to multivariate analysis using orthogonal partial least squares discriminant analysis (OPLS-DA).

Results 24 male and 13 female patients, 21 with alcohol-related cirrhosis, 5 viral hepatitis, 11 mixed/other causes, with median (range) age 57 (35-75) years made up the study cohort. Fifteen patients had no HE while 15 had grades $1-2$ and 7 grades 3-4. Median MELD score was 14 (4-32) and median arterial ammonia level $106(19-268 \mu \mathrm{mol} / \mathrm{L})$. Ammonia level correlated weakly with HE grade (Kendall's $\tau=0.238, p=0.040$ ) but not with MELD score $(\tau=0.010, p=0.118)$. No multivariate models using $\mathrm{HE}$ grade as a categorical (OPLSDA) or continuous (OPLS) variables resulted in validity (eg, OPLS model: $\mathrm{R} 2(\mathrm{Y})=0.489, \mathrm{Q} 2(\mathrm{Y})=-0.091$ ). A 2-component OPLS model using ammonia as a Y variable identified multiple metabolites correlating with arterial ammonia (overall R2 $(\mathrm{Y})=0.566, \quad \mathrm{Q} 2(\mathrm{Y})=0.394$, cross-validated ANOVA $\mathrm{p}=0.0008$ ). Metabolites associated with high ammonia levels included pyruvate, 3-hydroxybutyrate, glutamate, phenylalanine and an unassigned resonance at $2.43 \mathrm{ppm}$, while the resonances associated with lipoproteins correlated negatively with ammonia.

Conclusion Plasma ${ }^{1} \mathrm{H}$ NMR spectroscopy did not discriminate effectively between grades of $\mathrm{HE}$ in this cohort of patients with cirrhosis and overt HE. Multiple metabolites correlate with arterial ammonia level with some overlap from the metabolic pathways implicated in high MELD patients. Alternative metabolic profiling techniques such as mass spectroscopy may be required to assist in diagnosis in these patients.

Competing interests I Coltart: None declared, M McPhail Grant/Research Support from: Wellcome Trust, UK, E Want: None declared, K Veselkov: None declared, E Holmes: None declared, J Wendon: None declared, S Taylor-Robinson: None declared, W Bernal: None declared, D Shawcross: None declared.

\section{REFERENCE}

1. Amathieu R, et al. Metabolomic approach by $1 \mathrm{H}$ NMR spectroscopy of serum for the assessment of chronic liver failure in patients with cirrhosis. J Proteome Res 2011; 10:3239-45

\section{PTU-046 METABOLIC PROFILING OF PLASMA BY NMR SPECTROSCOPY ACCURATELY PREDICTS OUTCOME IN PATIENTS WITH DECOMPENSATED CIRRHOSIS AND ACUTE ON CHRONIC LIVER FAILURE}

doi:10.1136/gutjnl-2012-302514c.46

${ }^{1,2} \mathrm{M}$ J W McPhail, ${ }^{*} \mathrm{D}$ Shawcross, ${ }^{2}$ I Coltart, ${ }^{3} \mathrm{E}$ J Want, ${ }^{3} \mathrm{~K}$ Veselkov, ${ }^{1} \mathrm{M}$ Crossey,
${ }^{2} \mathrm{C}$ Willars, ${ }^{2} \mathrm{G}$ Auzinger, ${ }^{2} \mathrm{~J}$ O'Grady, ${ }^{2}$ W Bernal, ${ }^{3} \mathrm{E}$ Holmes, ${ }^{2} \mathrm{~J}$ A Wendon, ${ }^{1} \mathrm{~S} \mathrm{D}$ Taylor-
Robinson. ${ }^{1}$ Liver 8 Anti Viral Centre, Imperial College London, London, UK; ${ }^{2}$ Institute of
Liver Studies, Kings College Hospital, London, UK; ${ }^{3}$ Biomolecular Medicine, Imperial College London, London, UK

Introduction Acute-on-chronic liver failure is associated with a high mortality and difficulty in outcome prediction. Neither liver specific 